\title{
INDENTATION SiZE EFFECT OF Al2O3 \\ CERAMICS MADE BY COLD IsOSTATIC PREssing AND SLIP CAsting
}

\author{
CuRKoviC, L. \& LALIC, M.
}

Abstract: The behaviour of indentation size effect (ISE) in Vickers hardness for alumina ceramics made by cold isostatic pressing (CIP) and slip casting (SC) is compared. To explain the origin of the ISE in alumina ceramics, the three approaches is proposed: the traditional Meyer Law; proporcional specimen resistance (PSR) model and modified proporcional specimen resistance (PSR) model.

Key words: indentation size effect, hardness, alumina ceramics, modeling
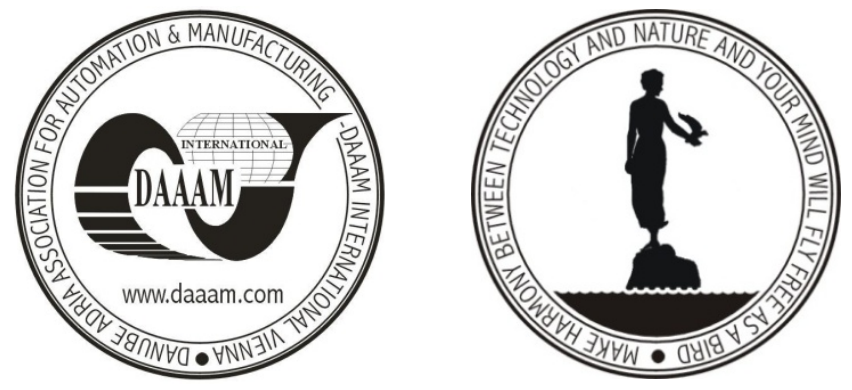

Authors' data: Prof. Curkovic, L[idija]; Lalic, M[arijo], Faculty of Mechanical Engineering and Naval Architecture, Ivana Lucica 5, 10000 Zagreb, Croatia, lidija.curkovic@fsb.hr, malalic@fssb.hr

This Publication has to be referred as: Curkovic, L[idija] \& L[alic], Marijo (2008). Indentation Size Effect of A12O3 Ceramics Made by Cold Isostatic Pressing and Slip Casting, Chapter 22 in DAAAM International Scientific Book 2008, pp. 249-256, B. Katalinic (Ed.), Published by DAAAM International, ISBN 978-3-901509-66-7, ISSN 1726-9687, Vienna, Austria

DOI: $10.2507 /$ daaam.scibook.2008.22 